\title{
O uso do cloridrato de piridoxina para induzir estro em cadelas
}

[The use of pyridoxine chloridrate to induce estrus in female dogs]

\author{
M. C. Silva, P.P.N. Snoeck*
}

Universidade Estadual de Santa Cruz - Ilhéus, BA

\begin{abstract}
RESUMO
Os agonistas dopaminérgicos são utilizados para induzir estro em cadelas, pois atuam na síntese e liberação de prolactina. Objetivou-se avaliar o efeito da piridoxina como indutor de estro em cadelas por agir na neurotransmissão dopaminérgica. Foram selecionadas 40 cadelas em anestro, divididas em quatro grupos experimentais, tratadas com $10 \mathrm{mg} / \mathrm{kg} / \mathrm{dia}(\mathrm{G} 1)$ e $50 \mathrm{mg} / \mathrm{kg} / \mathrm{dia}(\mathrm{G} 2)$ de cloridrato de piridoxina, $5 \mu \mathrm{g} / \mathrm{kg} / \mathrm{dia}$ (G3) de cabergolina e grupo controle/placebo (G4) por até 20 dias. Foram realizadas citologias vaginais a cada $24 \mathrm{~h}$ para acompanhamento do ciclo estral e análises hormonais (FSH, LH e PRL) no dia zero e 120h do início do tratamento. As cadelas do G3 (100\%) manifestaram proestro após 12 dias de tratamento aproximadamente, tempo inferior aos demais grupos $(\mathrm{P}<0,05)$. Apenas uma cadela do G1 e uma do G2 ficaram gestantes contra oito fêmeas do G3 e nenhuma do G4 $(\mathrm{P}<0,05)$. As concentrações plasmáticas de prolactina foram reduzidas nas fêmeas do G2 e G3 $(\mathrm{P}<0,05)$. As demais avaliações hormonais não sofreram influência do tratamento $(P>0,05)$. O cloridrato de piridoxina foi ineficiente para induzir estro em cadelas, mas foi capaz de suprimir a prolactina, de forma semelhante à cabergolina, quando utilizado na dose de $50 \mathrm{mg} / \mathrm{kg} / \mathrm{dia}$.
\end{abstract}

Palavras-chave: agonista dopaminérgico, anestro, prolactina, vitamina B6

\begin{abstract}
Dopaminergic agonists are used to induce estrus in female dogs as they act in the synthesis and release of prolactin. The objective of this study was to evaluate the effect of pyridoxine as an inducer of estrus by acting on dopaminergic neurotransmission. A total of 40 female dogs in anestrous were divided into four experimental groups treated with $10 \mathrm{mg} / \mathrm{kg} /$ day $(\mathrm{Gl})$ and $50 \mathrm{mg} / \mathrm{kg} /$ day $(\mathrm{G} 2)$ of pyridoxine hydrochloride, $5 \mu \mathrm{g} / \mathrm{kg} /$ day (G3) of cabergoline and control group/placebo (G4) for up to 20 days. Vaginal cytologies were performed every $24 \mathrm{~h}$ for follow-up of the estrous cycle and hormonal analyzes (FSH, LH and PRL) on day zero and 120 hours after the start of treatment. The female dogs from G3 (100\%) showed proestrus after 12 days of treatment, less time than the other groups $(P<0.05)$. Only one female from $G 1$ and one from $G 2$ were pregnant against eight from $G 3$ and none from $G 4(P<0.05)$. Plasma concentrations of prolactin were reduced by treatment in females from $G 2$ and $G 3(P<0.05)$. The other hormonal evaluations were not influenced by the treatment $(P>0.05)$. Pyridoxine chloridrate was inefficient to induce estrus in female dogs but was able to suppress prolactin when used at a dose of $50 \mathrm{mg} / \mathrm{kg} / \mathrm{day}$.
\end{abstract}

Keywords: dopaminergic agonist, anestrus, prolactin, vitamin B6

\section{INTRODUÇÃO}

As cadelas domésticas (Canis familiaris) são consideradas monoéstricas não sazonais de ovulação espontânea. Em média, apresentam dois ciclos ovulatórios por ano, seguidos por uma

Recebido em 8 de abril de 2019

Aceito em 9 de abril de 2019

* Autor para correspondência (corresponding author)

E-mail: paolasnoeck@gmail.com fase lútea com duração média de dois meses, mesmo na ausência de gestação (Sokolowski et al., 1977; Concannon, 2011; Kowalewski, 2017). O fim da fase luteínica é caracterizado pela perda da função luteal (luteólise) e, embora haja atividade no eixo hipotalâmico-hipofisáriogonadal, a responsividade dos ovários às 
gonadotrofinas é insuficiente para dar início a um próximo ciclo e o animal apresenta um estágio de inatividade sexual prolongado (Concannon, 2011; Kowalewski, 2017). A indução de estro tem sido utilizada como forma de melhorar a eficiência reprodutiva de cadelas. Os agonistas dopaminérgicos (ADs) são frequentemente usados para redução do intervalo interestro ou para induzir o estro em cadelas com anestro prolongado, por meio da inibição da síntese e/ou liberação de prolactina (PRL) (Concannon, 1993; Verstegen et al., 1999; Ajitkumar e Praseeda, 2010; Antonov e Georgiev, 2015).

A principal função da PRL em mamíferos é estimular e manter a lactação. No entanto, em cadelas, também possui atividade luteotrófica (Concannon, 1993) e desempenha um papel fundamental na fisiopatologia da pseudogestação (Gobello, 2000). Além disso, atua no controle da função ovariana, regulando os mecanismos de desencadeamento do estro (Concannon, 2011). A cabergolina é um agonista dopaminérgico ergolíneo comumente utilizado em cadelas em diferentes estágios do anestro com o intuito de reduzir o período de inatividade sexual mediado pela PRL (Onclin e Verstegen, 1997; Verstegen et al., 1999; Concannon, 1993; Ajitkumar e Praseeda, 2010; Nak et al., 2012; Shinde et al, 2014, Mogheiseh et al., 2017). Apresenta uma potente e prolongada atividade redutora de PRL com alta afinidade por receptores D2 e baixa afinidade para os receptores de serotonina (Muller e Nistico, 1989; Rains et al., 1995). Essas características permitem administração uma vez ao dia e com poucos efeitos colaterais, quando comparada a outros derivados de ergot, como a bromocriptina e a metergolina (Gobello, 2000; Antonov e Georgiev, 2015).

A administração oral de cabergolina na dose de $5 \mu \mathrm{g} / \mathrm{kg} / \mathrm{dia}$, durante aproximadamente duas semanas, pode ser usada para indução de estro em cadelas no anestro, com taxa de concepção satisfatória para diferentes raças e grupos etários (Verstegen et al., 1999; Ajitkumar e Praseeda, 2010; Nak et al., 2012; Shinde et al., 2014; Mogheiseh et al., 2017).

O cloridrato de piridoxina (vitamina B6) atua na síntese e secreção de PRL (Papavasiliou et al., 1972; Foukas, 1973; Marcus, 1975; Delitala et al., 1976; McIntosh, 1976) como cofator na descarboxilação de L-DOPA (levodopa) em dopamina no hipotálamo (Delitala et al., 1976; Bruice, 2006). Estudos realizados com mulheres lactantes tratadas com 200mg de piridoxina demonstraram que essa vitamina foi capaz de reduzir as concentrações de PRL e suprimir a lactação em 95\% dos casos (Foukas, 1973). De forma semelhante, ratas tratadas com injeções de vitamina B6 $(50 \mathrm{mg})$ também apresentaram redução nas concentrações de PRL (Harris et al., 1978), o que sugere um efeito dopaminérgico da piridoxina sobre o hipotálamo (Delitala et al., 1976). Em cães, a vitamina B6 pode ser utilizada na dose de $50 \mathrm{mg} / \mathrm{kg} / \mathrm{dia}$, por até 107 dias, sem sinais clínicos de toxicidade (Phillips et al., 1978). Doses mais elevadas $(150 \mathrm{mg} / \mathrm{kg} / \mathrm{dia})$ por um longo período de tratamento (100 a 120 dias) podem induzir quadros de neurotoxicidade subaguda, com sintomas de perda proprioceptiva, envolvendo ambos os lados e membros anteriores e posteriores, caracterizados por movimentos rígidos, espásticos e dismétricos (Hoover e Carlton, 1981).

O efeito da vitamina B6 sobre a supressão de PRL em cadelas bem como o seu efeito sobre o ciclo reprodutivo nessa espécie ainda não foram investigados. É possível que o cloridrato de piridoxina possua uma atividade dopaminérgica, influenciando nas concentrações séricas de PRL e, dessa forma, possa ser uma alternativa no auxílio do controle reprodutivo em cães, além de um agente potencial no tratamento da lactação na pseudociese. Dessa forma, buscou-se investigar o efeito do cloridrato de piridoxina na restauração de ciclos ovulatórios em cadelas no anestro, bem como sua influência sobre a concentração de prolactina nessa espécie.

\section{MATERIAL E MÉTODOS}

Foram selecionadas 40 cadelas da raça Bulldog francês, de dois canis distintos, com manejos semelhantes e localizados em mesma região. Os animais possuíam entre dois e seis anos de idade, com peso variando entre oito e 12 quilos, clinicamente saudáveis, vacinados e vermifugados. Durante o período experimental, os animais permaneceram alojados em baias com dois a três animais, com uma área coletiva de recreação e banho de sol. A alimentação era fornecida duas vezes ao dia, com ração comercial seca (Farmina Pet Foods ${ }^{\circledR}$ ), em quantidade 
adequada para manutenção do peso corporal, e água ad libitum.

Os animais foram divididos aleatoriamente em quatro grupos, cada um com 10 animais em estágio de anestro intermediário (130-160 dias de anestro) (Verstegen et al., 1999) e com concentração de progesterona sérica inferior a $1 \mathrm{ng} / \mathrm{mL}$. Os fármacos foram administrados diariamente, por via oral, em forma de cápsulas manipuladas (Vida Animal ${ }^{\circledR}$ ), até três dias após a manifestação do proestro ou por um período máximo de 20 dias. O grupo 1 (G1) recebeu cloridrato de piridoxina $10 \mathrm{mg} / \mathrm{kg} / \mathrm{dia}$; o grupo 2 (G2), cloridrato de piridoxina $50 \mathrm{mg} / \mathrm{kg} / \mathrm{dia}$; o grupo 3 (G3), cabergolina $5 \mu \mathrm{g} / \mathrm{kg} / \mathrm{dia}$; e o grupo controle (G4), placebo (cápsulas vazias).

O ciclo estral, durante o período de tratamento, foi acompanhado por meio de citologia vaginal feita a cada $72 \mathrm{~h}$, com o intuito de verificar o início do proestro, seguida de avaliações a cada 24h, para identificar o início do estro. As cadelas que responderam ao tratamento foram submetidas à inseminação artificial em fundo de vagina, com sêmen fresco $\left(350 \times 10^{6}\right.$ esptz progressivamente móveis/dose inseminante), quando apresentaram o índice de células superficiais anucleadas superior a $80 \%$ (Feldman e Nelson, 1987). As inseminações foram feitas a cada $48 \mathrm{~h}$ até o final do estro, definido pela alteração no padrão celular do epitélio vaginal e pela onda leucocitária.

O diagnóstico de gestação por ultrassonografia foi realizado 25 dias após a primeira inseminação, e as cadelas que apresentaram prenhez positiva foram acompanhadas até o parto para registro do tamanho da ninhada. As fêmeas que não responderam ao tratamento foram acompanhadas, semanalmente, por meio de citologia vaginal e pela manifestação de sintomas clínicos de estro (secreção vaginal sanguinolenta, edema vulvar e atração de machos) para registro do retorno da ciclicidade e definição do intervalo interestro.

Foram realizadas dosagens de prolactina, $\mathrm{LH}$ e FSH duas horas antes da administração dos fármacos (dia zero) e 120h após o início do tratamento, a fim de verificar possíveis estímulos à liberação de hormônios hipofisários por influência da piridoxina e/ou da cabergolina, uma vez que podem ocorrer alterações nas concentrações de prolactina a partir de 48h após administração oral de cabergolina (Verstegen et al., 1999). As análises foram realizadas no Instituto de Diagnóstico Animal (IDAN ${ }^{\circledR}$ ), com a utilização do método de quimioluminescência para avaliações de PRL, LH e FSH, e radioimunoensaio para análise de progesterona, realizadas na seleção dos animais para formação dos grupos experimentais. As amostras de sangue $(5 \mathrm{~mL})$ foram obtidas por punção venosa jugular em tubos de coleta de sangue a vácuo, com ativador de coágulo, centrifugadas a 3000rpm durante sete minutos. Alíquotas dessas amostras foram colocadas em tubos de microcentrífuga $(500 \mu \mathrm{L})$ e armazenadas a $-20^{\circ} \mathrm{C}$ até a realização das análises.

Para comparação dentro de um mesmo grupo, foi aplicado o teste de Wilcoxon para dados pareados. Para comparações antes e depois de cada tratamento entre os grupos, foi aplicado o teste de Kruskal-Wallis. Já para avaliar as informações relativas ao efeito do tratamento, foi aplicado $o$ teste de Kruskal-Wallis com desdobramentos via comparações múltiplas de Dunn em variáveis quantitativas, bem como o teste exato de Fisher para independência entre variáveis qualitativas (Milone, 2004; Lehmann e D'Abrera, 2006). As variáveis hormonais foram comparadas por meio de média, desvio- padrão e via teste de hipótese, ao nível de significância de $\mathrm{P}<0,05$. Este projeto foi aprovado pelo Comitê de Ética da Universidade Estadual de Santa Cruz (Ceua-UESC), sob o n ${ }^{\circ}$. 036/2015.

\section{RESULTADOS E DISCUSSÃO}

As cadelas tratadas com cabergolina (G3) manifestaram proestro após 12,3 $\pm 1,7$ dias de administração do fármaco, tempo inferior ao necessário para o início do proestro nas cadelas dos demais grupos $(\mathrm{P}<0,05)$. Todas as fêmeas do G3 apresentaram alteração no padrão celular na citologia vaginal esfoliativa, com aumento gradativo de células intermediárias pequenas e grandes. Também houve alterações físicas e/ou comportamentais, como hipertrofia e turgidez da vulva, descarga serossanguinolenta e atração de machos, em todas as cadelas tratadas com cabergolina. As fêmeas dos demais grupos experimentais sofreram variada influência na interrupção do repouso sexual, com duas do G1 (10mg de B6/kg/dia), três do G2 (50mg de B6/kg/dia) e uma do G4 (placebo) manifestando 
proestro/estro durante o período de tratamento, sendo que dessas apenas uma cadela do G1 e uma cadela do G2 apresentaram prenhez positiva $(\mathrm{P}<0,05)$.

Diversos fatores podem ter contribuído para a restauração do ciclo estral nas cadelas tratadas com piridoxina e placebo. É possível que a proximidade com outras fêmeas em estro (feromônios naturais) tenha colaborado para a interrupção do anestro por meio de estímulos sensoriais ou por interações sociais de dominância entre os grupos de fêmeas na presença do macho (Verstegen et al., 1999; Concannon, 2011). Além disso, o período correspondente ao anestro canino pode variar de 60 a 300 dias entre cadelas ou, até mesmo, para a mesma cadela em ciclos distintos (Sokolowski et al., 1977; Concannon, 1993; Concannon, 2011). As cadelas do experimento encontravam-se em anestro intermediário (130-160 dias de anestro), período em que algumas fêmeas podem interromper naturalmente o repouso sexual (Verstegen et al., 1999; Concannon, 2011).

A duração do período de proestro e do estro não variou entre as fêmeas de grupos experimentais distintos. No entanto, a cabergolina (G3) foi o único tratamento capaz de interferir $100 \%$ na atividade reprodutiva das cadelas (10 cadelas tratadas e 10 cadelas no estro), possibilitando o retorno à ciclicidade com $80 \%$ de prenhez positiva e 37 filhotes nascidos no total. O protocolo de tratamento com carbegolina $5 \mu \mathrm{g} / \mathrm{kg} / \mathrm{dia}$ demonstrou ser viável para indução de cios férteis em cadelas no anestro intermediário (130-160 dias de anestro), com custo médio do tratamento de 80,00 reais por animal com peso médio de 9,2kg (Tab. 1). O custo médio do tratamento com $10 \mathrm{e}$ $50 \mathrm{mg} / \mathrm{kg} / \mathrm{dia}$ de cloridrato de piridoxina ficou 25,60 e 38,10 reais por animal, respectivamente.

Tabela 1. Parâmetros reprodutivos associados à administração de cloridrato de piridoxina (10mg de B6/kg/dia ou 50mg de B6/kg/dia) ou cabergolina $(5 \mu \mathrm{g} / \mathrm{kg} / \mathrm{dia})$ em cadelas no anestro (130-160 dias de anestro)

\begin{tabular}{|c|c|c|c|c|}
\hline \multirow{2}{*}{ Parâmetros } & \multicolumn{4}{|l|}{ Tratamento } \\
\hline & G1 & G2 & G3 & G4 \\
\hline $\begin{array}{l}\text { Animais que responderam ao } \\
\text { tratamento }(\mathrm{n})\end{array}$ & $2 / 10^{\mathrm{a}}$ & $3 / 10^{\mathrm{a}}$ & $10 / 10^{b}$ & $1 / 10^{\mathrm{a}}$ \\
\hline Início do proestro (dias) & $19,9 \pm 0,3^{\mathrm{a}}$ & $19,8 \pm 0,4^{\mathrm{a}}$ & $12,3 \pm 1,7^{\mathrm{b}}$ & $20 \pm 0.0^{\mathrm{a}}$ \\
\hline Duração do proestro (dias) & $7,5 \pm 0,7$ & $7,7 \pm 0,6$ & $6,4 \pm 0,7$ & 8,0 \\
\hline Duração do estro (dias) & $8,5 \pm 0,7$ & $9,3 \pm 0,6$ & $7,9 \pm 0,6$ & 9,0 \\
\hline Cadelas gestantes (n) & $1^{\mathrm{a}}$ & $1^{\mathrm{a}}$ & $8^{\mathrm{b}}$ & $0^{\mathrm{a}}$ \\
\hline Total de filhotes (n) & $4^{\mathrm{a}}$ & $3^{\mathrm{a}}$ & $37^{\mathrm{b}}$ & $0 *$ \\
\hline Intervalo interestro (dias) & $195,0 \pm 8,0^{\mathrm{a}}$ & $194,0 \pm 5,0^{\mathrm{a}}$ & $169,0 \pm 7,0^{\mathrm{b}}$ & $197,0 \pm 9,0^{\mathrm{a}}$ \\
\hline
\end{tabular}

* Hiperplasia endometrial cística (achado ultrassonográfico).

${ }^{\mathrm{ab}}$ Letras diferentes na mesma linha indicam diferença significativa $(\mathrm{P}<0,05)$.

G1 (10mg de B6/kg/dia), G2 (50mg de B6/kg/dia), G3 (5 $\mathrm{gg}$ de cabergolina/kg/dia) e G4 (placebo).

$\mathrm{Na}$ avaliação ultrassonográfica, uma cadela do G4 apresentou inflamação uterina com acúmulo de exsudato, sem secreção vaginal (piometra fechada). Essa cadela foi submetida a tratamento com aglepristone, prostaglandina e doxiciclina, uma vez que as bactérias isoladas com maior frequência em piometras abertas e fechadas apresentam 84,6\% de sensibilidade à doxiciclina, sendo mais eficiente que outros fármacos antibióticos usados na rotina da clínica médica veterinária (Volpato et al., 2014). Após o tratamento, a cadela retornou à atividade reprodutiva, com gestação confirmada no estro subsequente.
A Tab. 2 mostra que, entre os hormônios estudados, a PRL foi o único hormônio que sofreu alteração com a utilização de cloridrato de piridoxina $(50 \mathrm{mg} / \mathrm{kg} /$ dia $)$ e cabergolina $(5 \mu \mathrm{g} / \mathrm{kg} / \mathrm{dia})$ após $120 \mathrm{~h}$ do início dos tratamentos, confirmando a ação inibitória desses fármacos na secreção de PRL em cadelas, sem interferência nas concentrações de FSH e LH. As diferentes respostas na indução do ciclo estral, portanto, podem ter sido influenciadas pelos distintos mecanismos de ação das moléculas de piridoxina e cabergolina, não dependendo exclusivamente da redução da PRL para interrupção do anestro. 
Tabela 2. Médias das concentrações plasmáticas de PRL, LH e FSH dosadas em cadelas (130-160 dias de anestro) no dia zero (dia do início do tratamento, antes da primeira administração dos fármacos) e $120 \mathrm{~h}$ após a administração de cloridrato de piridoxina ou cabergolina

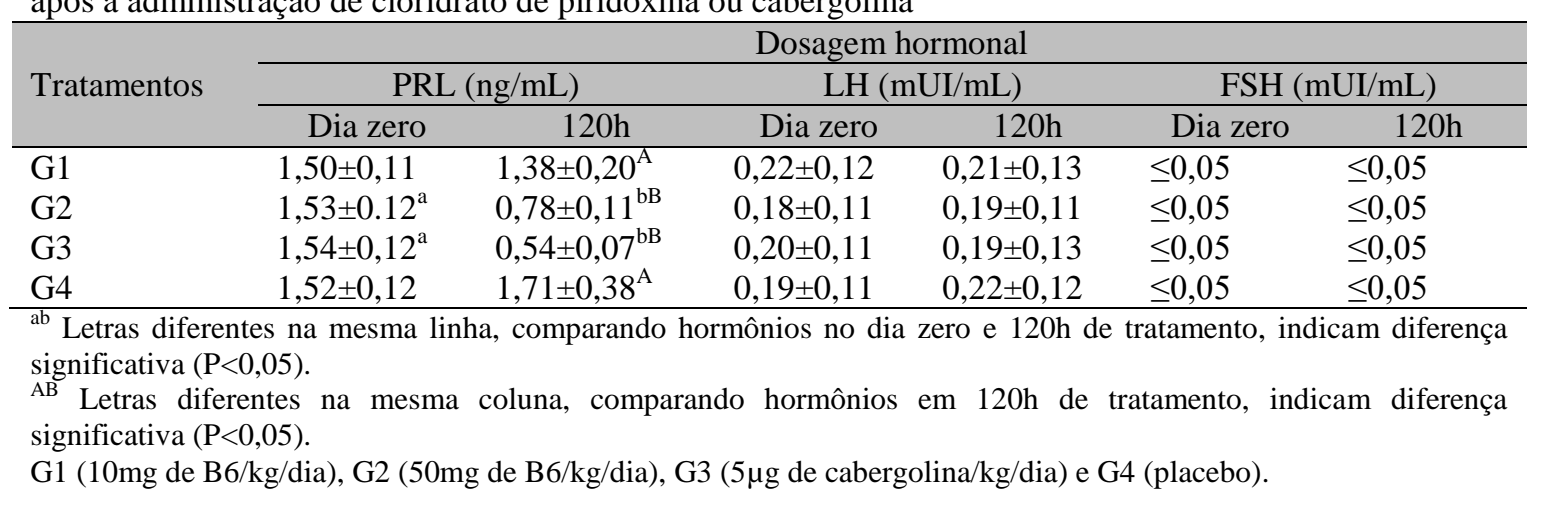

O cloridrato de piridoxina é uma coenzima capaz de catalisar reações de descarboxilação, interferindo na síntese de dopamina, neurotransmissor inibidor de PRL (Bruice, 2006). No entanto, a dopamina não ultrapassa a barreira hematoencefálica (BHE), sendo necessário que a L-DOPA, seu precursor, ultrapasse a BHE e sofra um processo de descarboxilação que requer o cofator fosfato de piridoxal, conhecido comercialmente como cloridrato de piridoxina, para que ocorra a conversão de L-DOPA em dopamina no hipotálamo (Bruice, 2006; Standaert e Galanter, 2009).

A cabergolina, por sua vez, aumenta a neurotransmissão dopaminérgica por meio do estímulo direto de receptores $\mathrm{D} 2$, com resposta mais estável que o cloridrato de piridoxina, uma vez que os ADs não competem com aminoácidos para que ocorra o seu transporte através da BHE e não necessitam de conversão enzimática. Dessa forma, os $\mathrm{ADs}$ permanecem efetivos por mais tempo, quando comparados à L-DOPA, sendo possível uma frequência de administração reduzida e resposta mais uniforme (Standaert e Galanter, 2009).

O estudo confirmou os efeitos inibitórios da vitamina B6 $(50 \mathrm{mg} / \mathrm{kg})$ sobre a PRL $(\mathrm{P}<0,05)$, após $120 \mathrm{~h}$ do início dos tratamentos, sem eficácia na indução de estro, demonstrando que a supressão da PRL não é suficiente para interromper o anestro em cadelas. É possível que diferentes mecanismos de ação entre as moléculas de piridoxina e cabergolina possam ter contribuído para uma resposta mais estável da cabergolina (Bruice, 2006; Standaert e Galanter,
2009), permitindo maior controle parácrino da foliculogênese. Nesse contexto, o hormônio antimülleriano (Nagashima et al., 2016), bem como o IGF-1, o hormônio do crescimento, a insulina, o FGF-2, o FGF-7 e a ativina, pode desempenhar um importante papel na transição de anestro para estro, atuando diretamente no desenvolvimento folicular em cães, sendo necessário aprofundar estudos com cultura folicular in vitro, para melhor compreensão dos controles parácrinos da reprodução em cães (Songsasen et al., 2017).

As cadelas tratadas com cabergolina apresentaram intervalo interestro mais curto do que as cadelas dos demais grupos estudados, confirmando que esse AD pode ser utilizado para antecipar o estro em cadelas no anestro intermediário (Vertegen et al., 1999). A indução de estro pela supressão de PRL neste estudo não foi influenciada pela ação luteotrófica da PRL, uma vez que, em todas as cadelas estudadas, as concentrações de progesterona eram basais no início do tratamento. Também parece improvável que a cabergolina e a vitamina B6 afetem diretamente os neurônios de $\mathrm{GnRH}$, visto que não foram observados efeitos sobre a concentração sérica de FSH e LH após 120h do início da administração dos fármacos, sendo necessárias novas avaliações, em diferentes tempos, devido ao padrão de secreção pulsátil desses hormônios em cadelas (Concannon, 1993).

Nenhuma cadela deste estudo manifestou efeitos colaterais adversos, durante os 20 dias de tratamento, com a administração de cloridrato de piridoxina ou cabergolina. Esses resultados demonstram que a vitamina B6 pode ser utilizada 
para reduzir as concentrações de PRL em cadelas, de forma semelhante à cabergolina, quando administrada na dose de $50 \mathrm{mg} / \mathrm{kg} / \mathrm{dia}$, sem sinais de toxicidade e sem influência sobre o ciclo estral. Outros estudos são necessários para investigar o efeito do cloridrato de piridoxina sobre a secreção láctea em cadelas.

\section{CONCLUSÃO}

O cloridrato de piridoxina nas concentrações estudadas não foi eficaz como a cabergolina para induzir o retorno da atividade cíclica e, portanto, não deve ser utilizado para protocolos de indução de estro em cadelas. Por sua vez, o tratamento com cloridrato de piridoxina $50 \mathrm{mg} / \mathrm{kg} / \mathrm{dia}$ foi capaz de atuar na supressão da prolactina em cadelas no anestro intermediário, assim como a cabergolina $5 \mu \mathrm{g} / \mathrm{kg} / \mathrm{dia}$, demonstrando uma possível ação seletiva do cloridrato de piridoxina sobre a PRL, uma vez que as concentrações de FSH e LH mantiveram-se inalteradas quando avaliadas $120 \mathrm{~h}$ após o início do tratamento. Esses resultados indicam que a vitamina B6 pode ser um potencial agente no tratamento de hiperprolactinemia em cadelas, necessitando de outros estudos para confirmar a sua ação sobre a interrupção da secreção láctea no pós-parto ou em estado de pseudogestação.

\section{AGRADECIMENTOS}

Agradecemos ao laboratório de análises clínicas $\mathrm{IDAN}^{\circledR}$, à farmácia de manipulação Vida Animal $^{\circledR}$, aos canis Stone Pet Center e Atlantic Valley, à equipe de médicos veterinários e auxiliares da Clínica Veterinária Pet do $\mathrm{Bem}^{\circledR} \mathrm{e}$ ao $\mathrm{CNPq}$, que colaboraram na execução das diversas etapas da realização deste trabalho.

\section{REFERÊNCIAS}

AJITKUMAR, G.; PRASEEDA, R. Induction of fertile oestrus in dogs using cabergoline. Vet. Scan. Online Ve.t J., v.5, p.56, 2010. Disponível $\mathrm{em}:<\mathrm{http}: / /$ vetscan.co.in/v5n1/induction_of_fertil e_oestrus_in_dogs_using_cabergoline.ht $>$. Acessado em: 26 abr. 2016.

ANTONOV, A.; GEORGIEV, P. Induction of estrus in bitches with the dopamine agonist bromocriptine. Int. J. Adv. Res., v.3, p.962-965, 2015.
BRUICE, P.Y. Química orgânica. São Paulo: Person Prentice Hall, 2006. v.2, 46p.

CONCANNON, P.W. Biology of gonadotrophin secretion in adult and prepubertal female dogs. $J$. Reprod. Fertil. Suppl., v.47, p.3-27, 1993.

CONCANNON, P.W. Reproductive cycles of the domestic bitch. Anim. Reprod. Sci., v.124, p.200210, 2011.

DELITALA, G.; MASAlA, A.; ALAGNA, S.; DEVILLA, L. Effect of pyridoxine on human hypophyseal trophic hormone release: a possible stimulation of hypothalamic dopaminergic pathway. J. Clin. Endocrinol. Metab., v.42, p.603-606, 1976.

FELDMAN, E.C.; NELSON, R.W. Canine female reproduction. In: FELDMAN, E.C.; NELSON, R.W. (Eds.). Canine and feline endocrinology and reproduction. Philadelphia: Elsevier, 1987, p.399-480.

FOUKAS, M.D. An antilactogenic effect of pyridoxine. J. Obstet. Gynaecol., v.80, p.718720, 1973.

GOBELlO, C. Aplicaciones clínicas de los agonistas dopaminérgicos en reproducción canina. Analec. Vet., v.20, p.31-38, 2000. Available in: <http://sedici.unlp.edu.ar/bitstream/handle/10915 /11121/documento_completo__.pdf?sequence $=1$ $>$. Accessed in: 04 May 2016.

HARRIS, A.R.C.; SMITH, M.S.; ALEX, S. et al. Pyridoxine (B6)-Induced inhibition of prolactin release in the female rat. Endocrinology, v.102, p362-366, 1978.

HOOVER, D.M.; CARLTON, W.W. The subacute neurotoxicity of excess pyridoxine $\mathrm{HCl}$ and clioquinol (5-chloro-7-iodo-8hydroxyquinoline) in beagle dogs. I. Clinical disease. Vet. Pathol., v.18, p.745-756, 1981.

KOWALEWSKI, M. Regulation of corpus luteum function in the domestic dog (canis familiaris) and comparative aspects of luteal function in the domestic cat (Felis catus). In: In: MEIDAN, R. (Ed.). The life cycle of the corpus luteum. Switzerland: Springer International Publishing, 2017. p.133-157.

LEHMANN, E.L.; D'ABRERA, H.J.M. Nonparametrics: statistical methods based on ranks. New York: Springer, 2006. 463p. 
MARCUS, R.G. Suppression of lactation with high doses of pyridoxine. S. Afr. Med. J., v.49, p.2155-2156, 1975.

MCINTOSH, E.N. Treatment of women with the galactorrhea-amenorrhea syndrome with pyridoxine (vitamin B6). J. Clin. Endocril. Metab., v.42, p.1192-1195, 1976.

MILONE, G. Estatística geral e aplicada. São Paulo: Atlas, 2004. 498p.

MOGHEISEH, A.; NAZIFI, S.; AHMADI, M.R. et al. Comparing the Vitex-castus extract and cabergoline effects on estrous induction in female dogs. Comp. Clin. Pathol., v.26,p.1-5, 2017.

MULLER, E.E.; NISTICÒ, G. Neurotransmitter regulation of the anterior pituitary. Brain messengers and the pituitary. San Diego: Academic Press, 1989. 404p.

NAGASHIMA, J.B.; HANSEN, B.S.; SONGSASEN, N. et al. Anti-müllerian hormone in the domestic dog during the anestrus to oestrous transition. Reprod. Domest. Anim., v.51, p.158-164, 2016.

NAK, D.; NAK, Y.; SIMSEK, G. Comparison of the use of cabergoline and gonadotrophin to treat primary and secondary anoestrus in bitches. Aust. Vet. J., v.90, p.194-196, 2012.

ONCLIN， K.; VERSTEGEN， J.P. In vivo investigation of luteal function in dogs: effects of cabergoline, a dopamine agonist, and prolactin on progesterone secretion during mid-pregnancy and-diestrus. Domest. Anim. Endocrinol., v.14, p.25-38, 1997.

PAPAVASILIOU, P.S.; COTZIAS, G.C.; DÜBY, S.E. et al. Levodopa in Parkinsonism: potentiation of central effects with a peripheral inhibitor. N. Engl. J. Med., v.286, p.8-14, 1972.

PHILLIPS, W.E.; MILLS, J.H.; CHARBONNEAU, S.M. et al. Subacute toxicity of pyridoxine hydrochloride in the beagle dog. Toxicol. Appl. Pharmacol., v.44, p.323-333, 1978.
RAINS, C.P.; BRYSON, H.M.; FITTON, A. Cabergoline: a review of its pharmacological properties and therapeutic potential in the treatment of hyperprolactinemia and inhibition of lactation. Drugs, v.49, p.255-279, 1995.

SHINDE, S.; KRISHNAPPA, B.; KUMAR, B. et al. Induction of fertile estrus in bitch (pug) with cabergolin-a case report. Int. J. Vet. Sci., v.3, p.1-3, 2014.

SOKOLOWSKI, J.H.; STOVER, D.G.; VANRAVENSWAAY, F. Seasonal incidence of estrus and interestrous interval for bitches of seven breeds. J. Am. Vet. Med. Assoc., v.171, p.271-273, 1977.

SONGSASEN, N.; NAGASHIMA, J.; THONGKITTIDILOK, C. Endocrine and paracrine controls of canine follicular development and function. Reprod. Domest. Anim., v.52, p.29-34, 2017.

STANDAERT, D.; GALANTER, J.M. Farmacologia da neurotransmissão dopaminérgica. Princípios de farmacologia: a base fisiopatologia da farmacoterapia. 2009. p.166-185. Disponível em:

<http://leg.ufpi.br/subsiteFiles/lapnex/arquivos/fi les/Farmacologia\%20da\%20neurotransmissao\%2 0dopaminergica.pdf>. Acessado em 10 jun. 2017.

VERSTEGEN, J.P.; ONCLIN, K.; SILVA, L.D.; CONCANNON, P.W. Effect of stage of anestrus on the induction of estrus by the dopamine agonist cabergoline in dogs. Theriogenology, v.51, p.597-611, 1999

VOLPATO, R.; ARAUJO, M.S.; TSUNEMI, M.H. et al. Caracterização microbiológica e perfil de resistência das bactérias isoladas do útero de cadelas com piometra aberta e fechada. Rev. Port. Cien. Vet., p.100-104, 2014. 